\title{
Electrically-Pumped Wavelength-Tunable GaAs Quantum Dots Interfaced with Rubidium Atoms
}

Huiying Huang, ${ }^{\dagger, \ddagger}$ Rinaldo Trotta, ${ }^{*},{ }^{\dagger}$ Yongheng Huo, ${ }^{*}, \dagger, \dagger$ Thomas Lettner, ${ }^{\dagger}$ Johannes S. Wildmann,

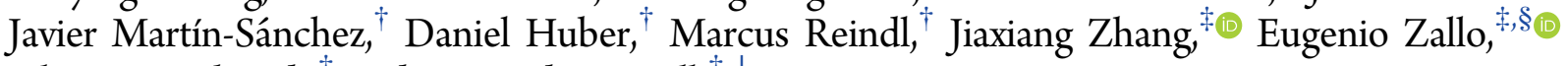
Oliver G. Schmidt, ${ }^{\dagger}$ and Armando Rastelli ${ }^{\dagger, \perp}$

${ }^{\dagger}$ Institute of Semiconductor and Solid State Physics, Johannes Kepler University Linz, Altenbergerstraße 69, Linz, 4040, Austria

${ }^{\ddagger}$ Institute for Integrative Nanosciences, IFW Dresden, Helmholtzstraße 20, Dresden, 01069 Germany

${ }^{\S}$ Paul-Drude-Institut für Festkörperelektronik Hausvogteiplatz 5-7, 10117 Berlin, Germany

${ }^{\perp}$ Johannes Kepler University Linz, Linz Institute of Technology (LIT), Altenbergerstraße 69, Linz, 4040, Austria

Supporting Information

ABSTRACT: We demonstrate the first wavelength-tunable electrically pumped source of nonclassical light that can emit photons with wavelength in resonance with the $\mathrm{D}_{2}$ transitions of ${ }^{87} \mathrm{Rb}$ atoms. The device is fabricated by integrating a novel GaAs single-quantum-dot light-emitting diode (LED) onto a piezoelectric actuator. By feeding the emitted photons into a $75 \mathrm{~mm}$ long cell containing warm ${ }^{87} \mathrm{Rb}$ vapor, we observe slow-light with a temporal delay of up to $3.4 \mathrm{~ns}$. In view of the possibility of using ${ }^{87} \mathrm{Rb}$ atomic vapors as quantum memories, this work makes an important step toward the realization of hybrid-quantum systems for future quantum networks.

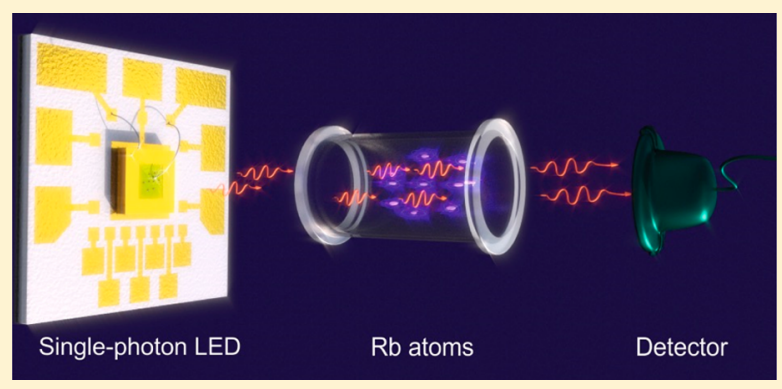

KEYWORDS: single photon source, GaAs quantum dots, light emitting diodes, strain actuators, atomic vapors

Sis ingle photon sources (SPSs) play a key role in quantum communication and quantum computation. ${ }^{1,2}$ For practical applications, it would be highly desirable to have electricallypumped SPSs that are compatible with other key elements of future quantum networks, such as quantum memories and single photon detectors.

Single semiconductor quantum dots (QDs), or "artificial atoms”, are arguably among the most promising SPSs because of their capability of emitting single photons with high efficiency, ${ }^{5}$ at high speed, ${ }^{6}$ and with a large degree of indistinguishability. ${ }^{7,8}$ Furthermore, semiconductor QDs can be embedded into the active region of light-emitting diodes, ${ }^{9-13}$ and their optical properties can be finely adjusted using external perturbations. ${ }^{14-17}$

The combination of artificial and natural atoms, a research avenue pioneered by Akopian et al., ${ }^{18}$ may allow storing the state of single photons emitted by a $\mathrm{QD}$ in atomic vapors or Bose-Einstein-Condensates. ${ }^{19}$ Such atomic systems offer, in fact, particularly long storage times ${ }^{3}$ and may become crucial ingredients for building up a quantum repeater. ${ }^{4,20}$ One of the key requirements to build up this hybrid artificial-natural atomic system is the availability of QDs with emission precisely tuned to atomic transitions. In the first work, ${ }^{18}$ focused on $\mathrm{GaAs} / \mathrm{AlGaAs}$ QDs interfaced with ${ }^{87} \mathrm{Rb}$ atomic vapors, energy tuning and $\mathrm{QD}$ excitation were achieved through magnetic fields and an external laser source, respectively. This makes the overall single-photon source extremely bulky and impractical for the envisioned hybrid quantum networks.
In this work, we report on the first realization of a wavelength-tunable GaAs QD based quantum-light-emitting diode (Q-LED), an all electrically driven source of single photons (see Figure 1) in which both the excitation and wavelength-tunability are provided on-chip while preserving the compatibility with the ${ }^{87} \mathrm{Rb}_{2}$ line transitions. To demonstrate the suitability of the device for future applications based on

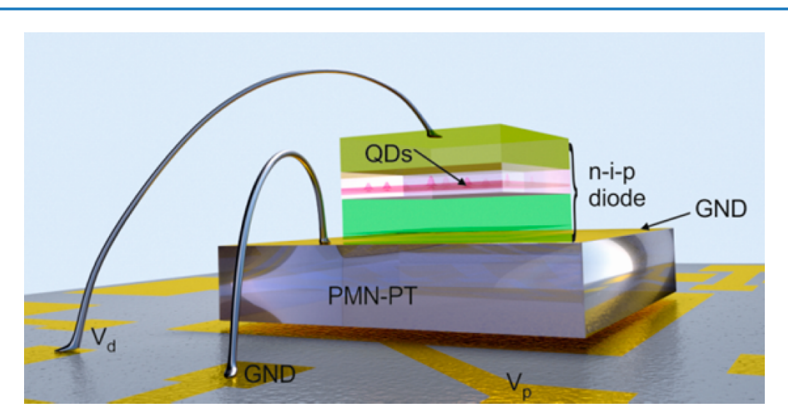

Figure 1. Sketch of the electrically pumped wavelength-tunable QLED. The n-i-p diode contains GaAs QDs embedded in $\mathrm{Al}_{0.4} \mathrm{Ga}_{0.6} \mathrm{As}$ barriers and is integrated on a PMN-PT piezoelectric actuator, which provides variable strain fields to tune the photon emission energy. $V_{\mathrm{d}}$ is the bias voltage applied to the diode and $V_{\mathrm{p}}$ is the voltage applied to the actuator.

Received: November 23, 2016

Published: March 15, 2017 
hybrid QD atomic systems, we send the emitted light into a ${ }^{87} \mathrm{Rb}$-vapor cell and observe slow single photons when the $\mathrm{QD}$ transition energy is fine-tuned through the hyperfine-split $\mathrm{D}_{2}$ lines of $\mathrm{Rb}$.

The wavelength-tunable Q-LED is obtained by integrating nanomembrane $\mathrm{p}-\mathrm{i}-\mathrm{n}$ diodes on a $\left[\mathrm{Pb}\left(\mathrm{Mg}_{1 / 3} \mathrm{Nb}_{2 / 3}\right) \mathrm{O}_{3}\right]_{0.72^{-}}$ $\left[\mathrm{PbTiO}_{3}\right]_{0.28}(\mathrm{PMN}-\mathrm{PT})$ piezoelectric actuator, as sketched in Figure 1. It is important to note that, while single-QD-LEDs have been reported for other material systems, such as InGaAs/ $\mathrm{GaAs},{ }^{6,9-11,13}$ and $\mathrm{InP} / \mathrm{InGaP},{ }^{12}$ reports on electrically controlled GaAs QDs are limited to n-i-Schottky structures, ${ }^{21,22}$ which are unsuitable for electrical injection of both electrons and holes. The Q-LED nanomembrane used here was grown by molecular beam epitaxy (MBE) and consists of a QD layer in the middle of a $124 \mathrm{~nm} \mathrm{Al}_{0.4} \mathrm{Ga}_{0.6} \mathrm{As}$ intrinsic layer, which is sandwiched between a $160 \mathrm{~nm}$ n-doped $\mathrm{Al}_{0.4} \mathrm{Ga}_{0.6}$ As layer and an $85 \mathrm{~nm}$ p-doped $\mathrm{Al}_{0.4} \mathrm{Ga}_{0.6}$ As layer, see Figure 1. The QDs were obtained by an optimized local-droplet-etching approach consisting in Al-droplet etching ${ }^{23}$ of an $\mathrm{Al}_{0.4} \mathrm{Ga}_{0.6}$ As layer followed by nanohole filling with GaAs. ${ }^{24,25}$ By adjusting the GaAs filling amount, the QD emission wavelength can be tuned to be around $780 \mathrm{~nm},{ }^{26}$ close to the ${ }^{87} \mathrm{Rb} \mathrm{D}_{2}$ transitions. The heterostructure was capped by $10 \mathrm{~nm}$ highly doped GaAs on both sides to prevent oxidation and to enable ohmic contacts. QDs grown with this technique can have a density lower than $10^{8} \mathrm{~cm}^{-2}$, making it easy to address single dots in microelectroluminescence ( $\mu$-EL) measurements. Recent studies ${ }^{27,28}$ have also shown that such QDs can emit highly indistinguishable and entangled photons, making them appealing for envisioned quantum-optical experiments and applications.

The active structure was grown on a sacrificial layer, which allows free-standing membranes to be fabricated and integrated onto a $300 \mu \mathrm{m}$ thick PMN-PT piezoelectric actuator via flipchip and gold thermocompression bonding. ${ }^{13}$ The device was mounted onto an AlN chip carrier providing electrical contacts both to the diode nanomembranes and the piezoelectric actuator. The current is injected into the Q-LED by applying a bias voltage $V_{\mathrm{d}}$ above the turn-on voltage of the diode, while the strain-state of the membrane is varied by applying a voltage $V_{\mathrm{p}}$ across the PMN-PT piezoelectric actuator, as illustrated in Figure 1. The two circuits share a common ground and allow independent control of LED current and, thus, emission intensity and strain, which in turn controls the emission wavelength due to strain-induced effects. ${ }^{6,13,16,17,29}$ The thickness of the layers and the position of the QD is chosen to obtain a simple Au-semiconductor-air planar cavity, which enhances the collection efficiency of the QD emission. ${ }^{6,13}$

In order to measure the EL spectra of the single Q-LED, the device is mounted on the coldfinger of a helium-flow cryostat and cooled to temperatures of $\sim 10 \mathrm{~K}$. Figure 2a displays a typical $I-V$ trace of the Q-LED, showing no observable current below the turn-on voltage and a rapid increase beyond it, as in standard LEDs. (Note that the sign of the voltage needed to obtain EL is negative, since the top contact of the diode is ntype. In Figure 2 and in the following we omit the minus sign on both the voltage and the current.) To collect EL from single QDs, a 50X microscope objective with a numerical aperture of 0.42 is used. The signal is then guided into a spectrometer equipped with an 1800 lines/mm grating and a liquid-nitrogencooled Si-CCD. The spectrometer has a resolution of about 40 $\mu \mathrm{eV}$ at the wavelength of $780 \mathrm{~nm}$. In Figure $2 \mathrm{~b}$, we show the bias-voltage dependent $\mu$-EL map from a single QD. When the bias voltage $V_{\mathrm{d}}$ exceeds the turn-on voltage of the diode (about (a)
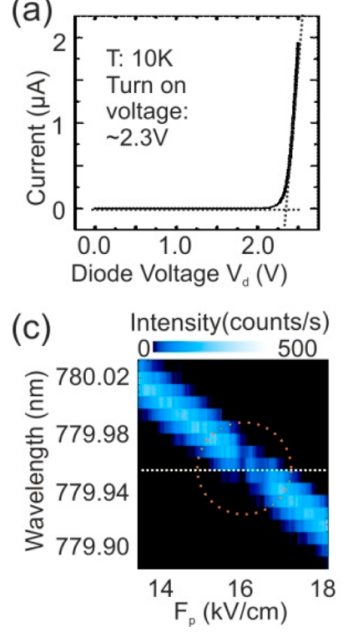

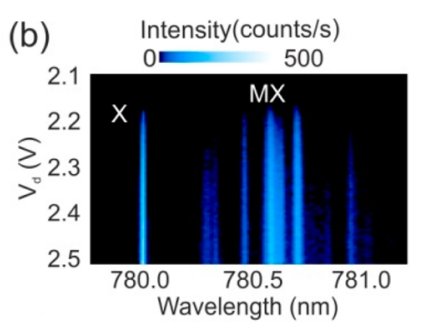

(d)

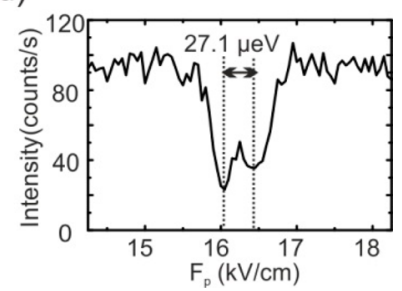

Figure 2. (a) Typical current-voltage $(I-V)$ characteristics of the QD LED, with a diode turn-on voltage of $\sim 2.3 \mathrm{~V}$. (b) Evolution of the electroluminescence (EL) spectra of a single QD embedded in the tunable-LED with the magnitude of the applied voltage $V_{\mathrm{d}}$. The intensity (in CCD counts per second) is color-coded. In the spectra, the brightest line stems from the neutral exciton $(X)$ transition, which is well separated from the group of low-energy states, which are ascribed to charged and neutral multiexcitonic (MX) states. (c) Colorcoded microelectroluminescence ( $\mu$-EL) spectra of the exciton in (a), whose wavelength is scanned across the $\mathrm{D}_{2}$ transitions of the ${ }^{87} \mathrm{Rb}$ cloud (at a temperature $T_{\mathrm{Rb}}=70{ }^{\circ} \mathrm{C}$ ) by applying variable stress on the Q-LED membrane. The white dotted line indicates the middle wavelength of the $\mathrm{D}_{2}$ transitions. The transmitted intensity drops at $16.05 \mathrm{kV} / \mathrm{cm}$ and $16.40 \mathrm{kV} / \mathrm{cm}$ due to the absorption by the atomic vapor (region indicated by dashed circle). (d) Transmitted intensity as a function of the electric field applied to the piezoelectric actuator $\left(F_{p}\right)$, obtained by extracting the peak intensity values from the corresponding spectra in (c), as described in the text. The two dips correspond to the two hyperfine lines of the ground state of the $\mathrm{D}_{2}$ transitions.

2.2-2.3 V), photons of different wavelengths are emitted due to the recombination processes of the injected electrons and holes. The line width of the dominant neutral exciton state $(X)$ is below the resolution limit of the setup. It is important to note that in the explored range of $V_{\mathrm{d}}$ only the line intensity changes, while no appreciable spectral shift nor line-broadening is observed (see Figure 2b).

To interface QD photons with Rubidium atoms, we insert a $\mathrm{Rb}$ gas cell in the optical path, between the objective and the spectrometer. The cell, made of quartz and with a length of 75 $\mathrm{mm}$, contains mostly ${ }^{87} \mathrm{Rb}$ atoms, with some residual of ${ }^{85} \mathrm{Rb}$ atoms (estimated to be $\sim 4 \%$, see Supporting Information). A heating setup is used to change the vapor density of the cell. A temperature calibration of the heating setup can be found in the Supporting Information. As in ref 18, we use the double absorption resonance scheme to demonstrate slow single photons. ${ }^{3,18,30}$ Specifically, we tune the energy of the photons in the spectral range of the $\mathrm{D}_{2}$ transitions $\left(5 \mathrm{~S}_{1 / 2}\right.$ to $\left.5 \mathrm{P}_{3 / 2}\right)$ of ${ }^{87} \mathrm{Rb}(\sim 780 \mathrm{~nm})$, which consist of six lines in total. ${ }^{31}$ However, limited by the line width of the QD emission, the Doppler broadening of the atomic lines, and the spectrometer resolution, we can resolve only the two subgroups separated by the hyperfine splitting of the ground state $5 S_{1 / 2}$ of about 28 $\mu \mathrm{eV}$.

The emission wavelength of the neutral exciton $X$ (the dominant exciton's transition in Figure $2 b$ ) is scanned through 
the $\mathrm{D}_{2}$ transitions by gradually varying the electric field applied to the PMN-PT piezoelectric actuator, as shown in the colorcoded $\mu$-EL spectra of Figure 2c. Two transmission dips are clearly observed at $F_{\mathrm{p}}=16.05$ and $16.40 \mathrm{kV} / \mathrm{cm}$ due to the absorption at the $\mathrm{D}_{2}$ transitions. To better observe this effect, we extract from each spectrum the peak intensity (without background) of the $X$ line through a Lorentzian fit. The result is shown in Figure 2d, where the two intensity dips corresponding to the hyperfine splitting of the ground state are clearly identified. Please note that, even at these two dips, the transmission intensity is not zero, mostly due to the finite line width of the $X$ emission. The energy difference between the two dips is found to be $\sim 27 \mu \mathrm{eV}$, thus, matching the expectations. $^{31}$

When the emission energy of the photons emitted by the QLED is set to the range of the absorption doublet, we expect a temporal delay for the transmitted photons due to the strongly dispersive behavior of the vapor medium, ${ }^{30,32,33}$ which in turn leads to a decrease of the light group velocity, as discussed in the Supporting Information.

To observe such a delay and prove the nonclassical nature of the light emitted by our GaAs QD-LED, we measured the second-order autocorrelation function $g^{(2)}(\tau)$ of photons emitted by the $X$ recombination of another, brighter, QD using a Hanbury-Brown-Twiss (HBT) setup. This $X$ transition (see Figure 3a) consists of two orthogonally polarized lines separated by a fine-structure-splitting of $145 \mu \mathrm{eV}$. Only the brightest polarization component, with a line width of $58 \mu \mathrm{eV}$ is

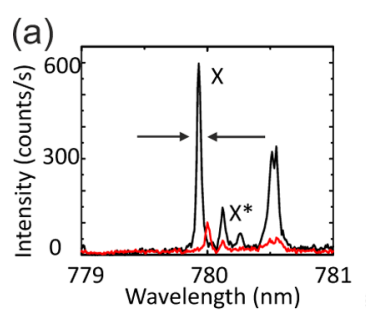

(b)

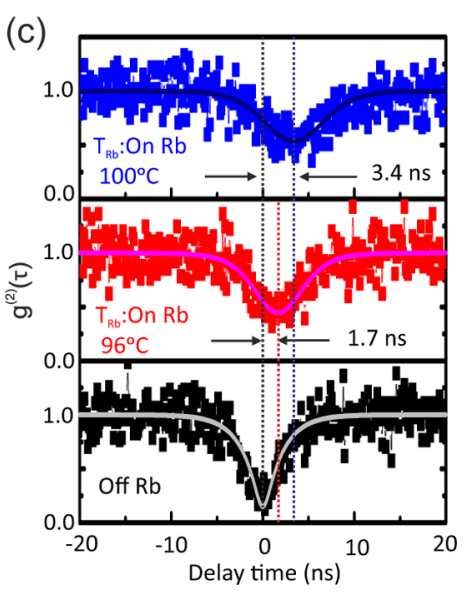

Figure 3. (a) EL spectra of a second QD, acquired with a double spectrometer equipped with two $1200 \mathrm{l} / \mathrm{mm}$ gratings (spectral resolution $\sim 30 \mu \mathrm{eV}$ ). The two spectra correspond to two orthogonal polarization directions. Only the bright line marked by arrows (with a line width of $58 \mu \mathrm{eV}$ ) is used in the following measurement. (b) HBT setup used to extract the second-order correlation function, $\mathrm{g}^{(2)}(\tau)$, of the EL emitted by a single QD embedded in the Q-LED. A $75 \mathrm{~mm}$ long ${ }^{87} \mathrm{Rb}$ vapor cell is inserted in one of the arms of the setup. (c) Black: $\mathrm{g}^{(2)}(\tau)$ measurement of the EL emission when the photon energy is tuned off resonance with respect to the ${ }^{87} \mathrm{Rb} \mathrm{D}_{2}$ transitions. Red and blue: $\mathrm{g}^{(2)}(\tau)$ values when the photon energy is tuned on resonance with the ${ }^{87} \mathrm{Rb} \mathrm{D}_{2}$ lines and the $\mathrm{Rb}$ cell temperature is 96 (red), and $100{ }^{\circ} \mathrm{C}$ (blue). The corresponding minimum values of $\mathrm{g}^{(2)}(\tau)$, reached at a $T_{\mathrm{Rb}}$-dependent delay time $\tau_{\mathrm{c}}\left(T_{\mathrm{Rb}}\right)$ are $0.15,0.44$, and 0.53 for the data displayed in of the black, red, and blue, respectively. The gradual increase of $\tau_{\mathrm{c}}$ and of $\mathrm{g}^{(2)}\left(\tau_{\mathrm{c}}\right)$ with increasing $T_{\mathrm{Rb}}$ comes from the dispersion of the atomic optical medium. This leads to slow light and, due to the finite line width of our photon source, to time-jitter and consequent broadening of the $\mathrm{g}^{(2)}(\tau)$ curve. selected for the HBT measurements. As sketched in Figure $3 \mathrm{~b}$, the $\mu$-EL beam is separated by a 50/50 beam splitter and sent to two avalanche photodiodes (APDs) via the two arms of the HBT setup. The time-resolution of the setup is about $1 \mathrm{~ns}$, as determined from an autocorrelation measurement of femtosecond laser pulses. In one of the paths, we placed the ${ }^{87} \mathrm{Rb}$ cell. Finally, the two APDs outputs are connected to correlation electronics to record coincidence counts. Results (with no background correction) are shown in Figure 3c. The black lines in Figure $3 c$ show the measured $g^{(2)}(\tau)$ when the QD-photons are tuned off resonance with respect the ${ }^{87} \mathrm{Rb} \mathrm{D}_{2}$ transitions. The second-order correlation function at zero time delay $g^{(2)}(0)$ reaches a value of 0.15 . The red and blue lines show the $\mathrm{g}^{(2)}$ results when the emission wavelength is turned on resonance with the $\mathrm{D}_{2}$ transitions at $\mathrm{Rb}$ cell temperatures $T_{\mathrm{Rb}}$ of 96 (red) and $100{ }^{\circ} \mathrm{C}$ (blue). Compared with the offresonance autocorrelation result, we observe two effects with increasing $T_{\mathrm{Rb}}$ : (1) the position of the minimum of the $\mathrm{g}^{(2)}(\tau)$, $\tau_{\mathcal{c}}$ shifts from 0 to $1.7 \mathrm{~ns}\left(\right.$ at $T_{\mathrm{Rb}}=96{ }^{\circ} \mathrm{C}$ ) and $3.4 \mathrm{~ns}\left(\right.$ at $T_{\mathrm{Rb}}=$ $\left.100{ }^{\circ} \mathrm{C}\right)$; (2) The values of $\mathrm{g}^{(2)}\left(\tau_{\mathrm{c}}\right)$ increase from 0.15 to 0.44 (at $T_{\mathrm{Rb}}=96{ }^{\circ} \mathrm{C}$ ) and $0.53\left(\right.$ at $T_{\mathrm{Rb}}=100{ }^{\circ} \mathrm{C}$ ), as indicated in Figure $3 \mathrm{c}$.

The dips in all three measurements clearly indicate that our device is capable of emitting nonclassical states of light. The temporal shifts of the minima of $\mathrm{g}^{(2)}(\tau)$ in resonant conditions can be easily explained by the reduction of group velocity of photons tuned between the absorption lines of $\mathrm{Rb}^{34}$ The increase of $\mathrm{g}^{(2)}\left(\tau_{\mathrm{c}}\right)$ is attributed to the strong optical dispersion of the atomic medium (which contains also some undesired ${ }^{85} \mathrm{Rb}$ ) combined with the finite line width of the QD emission $(58 \mu \mathrm{eV}) .^{33,35}$ More precisely, since the group velocity is wavelength-dependent, QD photons of slightly different energy traverse the vapor with different group velocities and thus escape the gas cell at slightly different times. In turn, the nonideal line width of the $\mathrm{X}$ emission of the selected $\mathrm{QD}$ is attributed to spectral jitter produced by fluctuating electric fields at the QD position. This spectral jitter, combined with the spectral dependence of the group velocity, leads to a time jitter in the arrival times of photons on the APDs. This time jitter (which increases with increasing cell temperature $T_{\mathrm{Rb}}$ ) adds up to the instrumental jitter of the APDs leading to a broadening and smearing of the $\mathrm{g}^{(2)}(\tau)$ dip. We model the dip position shifts by introducing a temporal shift $\tau_{\mathrm{c}}$ in the formula for the ideal second order correlation function of a two level system:

$$
\mathrm{g}^{(2)}(\tau)=1-\exp \left(\frac{\left|\tau-\tau_{\mathrm{c}}\right|}{t}\right)
$$

where $t$ accounts for exciton relaxation and decay rates. In the equation above we have assumed a negligibly small electronhole pair generation rate. ${ }^{36}$

To take into account the effect of time-jitter we perform a convolution with a Gaussian function

$$
G(\tau)=\left[w \sqrt{\frac{\pi}{4 \ln (2)}}\right]^{-1} \exp \left(-\frac{4 \ln (2) \tau^{2}}{w^{2}}\right)
$$

whose fwhm $w$ takes into account both the finite time resolution of the single photon detectors $(\Delta t)$ and the time jitter introduced by the dispersive atomic medium $\left(\Delta t_{\mathrm{c}}\right)$. By assuming Gaussian statistics and considering that these two jitter mechanisms are uncorrelated, we have $w^{2}=\Delta t^{2}+\Delta t_{c}^{2}$. 
After convolution, the equation describing the data shown in Figure $2 \mathrm{c}$ becomes

$$
F(\tau)=\mathrm{g}^{(2)}(\tau) * G(\tau)
$$

where $*$ indicates the convolution operation.

To reproduce the experimental data in the absence of the gas cell (or for QD emission far detuned from the atomic transitions), only the decay rate $t$ is used as free parameter (since $\Delta t$ is fixed at the experimental value, $\Delta t_{\mathrm{c}}=0$ and $\tau_{\mathrm{c}}=0$ ). The smooth curves in Figure $3 \mathrm{c}$ show, for each $\mathrm{g}^{(2)}(\tau)$ measurement, the corresponding fitting results. The decay time is found to be $t=2.0 \mathrm{~ns}$, an extremely long value that we attribute to slow relaxation dynamics of carriers from the high energy states (see ref 27). In the presence of the $\mathrm{Rb}$ cell at 96 ${ }^{\circ} \mathrm{C}$, a temperature-dependent temporal shift of $\tau_{\mathrm{c}}\left(96^{\circ} \mathrm{C}\right)=1.7$ ns, time jitter of $\Delta t_{\mathrm{c}}\left(96^{\circ} \mathrm{C}\right)=4.1 \mathrm{~ns}$ are instead needed to reproduce the experimental data. At $T_{\mathrm{Rb}}=100{ }^{\circ} \mathrm{C}$, the values are $\tau_{\mathrm{c}}\left(100{ }^{\circ} \mathrm{C}\right)=3.4 \mathrm{~ns}$ and $\Delta t_{\mathrm{c}}\left(100{ }^{\circ} \mathrm{C}\right)=5.7 \mathrm{~ns}$. It is worth emphasizing that the increased value of the $\mathrm{g}^{(2)}\left(\tau_{\mathrm{c}}\right)$ cannot be accounted for, as expected, by any artificial change in $t$, but it is totally ascribable to the additional time jitter $\Delta t_{\mathrm{c}}$ discussed above.

In conclusion, we have reported on the first quantum light emitting diode based on single GaAs/AlGaAs QDs and demonstrated its operation as an energy-tunable source of photons in the spectral range of the ${ }^{87} \mathrm{Rb} \mathrm{D}_{2}$ transitions. Autocorrelation measurements under continuous excitation clearly demonstrate antibunched emission, which is typical for single-photon sources. By comparing autocorrelation measurements with emission on/off resonance with the ${ }^{87} \mathrm{Rb} \mathrm{D}_{2}$ transitions we deduce that a double-resonance slow-light mechanism produces temporal delays of up to $3.4 \mathrm{~ns}$. To further increase the delay while keeping losses low (see ref 18), an isotopically purer $\mathrm{Rb}$ cell and quantum dot samples with even narrower emission lines would be required. One of the possible ways to improve the emission properties while keeping electrical excitation is to embed the QDs into resonanttunneling QD-LEDs, ${ }^{37}$ in which the electrons and holes are injected directly in the lowest energy levels. By using this method, we expect to be able to (i) reduce charge fluctuations in the QD surroundings, which produce inhomogeneous line broadening, and (ii) avoid carrier relaxation through the excited states, which leads to an undesired time-jitter in the photon emission. In addition, improvement of light extraction efficiency may be possible by proper engineering of the photonic environment. ${ }^{38}$ Finally, it should be noted that the slow light approach followed here is not sufficient for a quantum memory. Alternative approaches to store the photons emitted by our GaAs QD-LEDs may rather rely on electromagnetic induced transparency or Raman interactions in warm atomic clouds. ${ }^{39}$ We envision that our all-electrically controlled single photon LED, once integrated with chip-scale atom quantum memory, such as hollow-core fiber cell, ${ }^{40,41}$ may pave the way toward large-scale small volume hybrid quantum systems that can be used in future quantum networks.

\section{ASSOCIATED CONTENT}

\section{S Supporting Information}

The Supporting Information is available free of charge on the ACS Publications website at DOI: 10.1021/acsphotonics.6b00935.

Supporting figures and experimental details (PDF).

\section{AUTHOR INFORMATION}

\section{Corresponding Authors}

*E-mail: rinaldo.trotta@jku.at.

*E-mail: yongheng.huo@jku.at.

ORCID

Huiying Huang: 0000-0002-8946-8472 Jiaxiang Zhang: 0000-0002-8954-9256 Eugenio Zallo: 0000-0003-1796-9777

Funding

This work is financially supported by BMBF QuaHL-Rep (Contract Nos. 01BQ1032 and 01BQ1034), the European Union Seventh Framework Program 209 (FP7/2007-2013), under Grant Agreement No. 601126210 (HANAS), by the Austrian Science Fund (FWF), under Grant No. P29603, as well as by the European Research Council (ERC) under the European Union's Horizon 2020 research and innovation programme (SPQRel, grant agreement No. 679183).

\section{Notes}

The authors declare no competing financial interest.

\section{ACKNOWLEDGMENTS}

The authors thank B. Eichler, R. Engelhard, S. Harazim, F. Binder, A. Halliovic, U. Kainz, E. Vorhauer, and S. Bräuer for technical assistance and P. Atkinson, B. Höfer, V. Mahalingam, and F. Scholz for helpful discussions.

\section{REFERENCES}

(1) Pan, J. W.; Chen, Z. B.; Lu, C. Y.; Weinfurter, H.; Zeilinger, A.; Żukowski, M. Multiphoton entanglement and interferometry. Rev. Mod. Phys. 2012, 84 (2), 777-838.

(2) Aharonovich, I.; Englund, D.; Toth, M. Solid-state single-photon emitters. Nat. Photonics 2016, 10 (10), 631-641.

(3) Specht, H. P.; Nölleke, C.; Reiserer, A.; Uphoff, M.; Figueroa, E.; Ritter, S.; Rempe, G. A. A single-atom quantum memory. Nature 2011, 473 (7346), 190-193.

(4) Shields, A. J. Semiconductor quantum light sources. Nat. Photonics 2007, 1 (4), 215-223.

(5) Claudon, J.; Bleuse, J.; Malik, N. S.; Bazin, M.; Jaffrennou, P.; Gregersen, N.; Sauvan, C.; Lalanne, P.; Gérard, J.-M. A highly efficient single-photon source based on a quantum dot in a photonic nanowire. Nat. Photonics 2010, 4, 174-177.

(6) Zhang, J.; Ding, F.; Zallo, E.; Trotta, R.; Höfer, B.; Han, L.; Kumar, S.; Huo, Y.; Rastelli, A.; Schmidt, O. G. A nanomembranebased wavelength-tunable high-speed single-photon-emitting diode. Nano Lett. 2013, 13 (12), 5808-5813.

(7) Somaschi, N.; Giesz, V.; De Santis, L.; Loredo, J. C.; Almeida, M. P.; Hornecker, G.; Portalupi, S. L.; Grange, T.; Antón, C.; Demory, J.; Gómez, C.; Sagnes, I.; Lanzillotti-Kimura, N. D.; Lemaitre, A.; Auffeves, A.; White, A. G.; Lanco, L.; Senellart, P. Near-optimal singlephoton sources in the solid state. Nat. Photonics 2016, 10 (5), 340345.

(8) Ding, X.; He, Y.; Duan, Z. C.; Gregersen, N.; Chen, M. C.; Unsleber, S.; Maier, S.; Schneider, C.; Kamp, M.; Höfling, S.; Lu, C. Y.; Pan, J. W. On-demand single photons with high extraction efficiency and near-unity indistinguishability from a resonantly driven quantum dot in a micropillar. Phys. Rev. Lett. 2016, 116, 20401.

(9) Yuan, Z.; Kardynal, B. E.; Stevenson, R. M.; Shields, A. J.; Lobo, C. J.; Cooper, K.; Beattie, N. S.; Ritchie, D. A.; Pepper, M. Science (Washington, DC, U. S.) 2002, 295 (5552), 102-105.

(10) Reitzenstein, S.; Heindel, T.; Kistner, C.; Albert, F.; Braun, T.; Hopfmann, C.; Mrowinski, P.; Lermer, M.; Schneider, C.; Höfling, S.; Kamp, M.; Forchel, A. Electrically driven quantum dot micropillar light sources. IEEE J. Sel. Top. Quantum Electron. 2011, 17 (6), 1670-1680.

(11) Chung, T. H.; Juska, G.; Moroni, S. T.; Pescaglini, A.; Gocalinska, A.; Pelucchi, E. Selective carrier injection into patterned 
arrays of pyramidal quantum dots for entangled photon light-emitting diodes. Nat. Photonics 2016, 10, 203.

(12) Hargart, F.; Kessler, C. A.; Schwarzbäck, T.; Koroknay, E.; Weidenfeld, S.; Jetter, M.; Michler, P. Electrically driven quantum dot single-photon source at $2 \mathrm{GHz}$ excitation repetition rate with ultra-low emission time jitter. Appl. Phys. Lett. 2013, 102 (1), 11126.

(13) Trotta, R.; Atkinson, P.; Plumhof, J. D.; Zallo, E.; Rezaev, R. O.; Kumar, S.; Baunack, S.; Schröter, J. R.; Rastelli, A.; Schmidt, O. G. Nanomembrane Quantum-Light-Emitting Diodes Integrated onto Piezoelectric Actuators. Adv. Mater. 2012, 24 (20), 2668-2672.

(14) Trotta, R.; Zallo, E.; Ortix, C.; Atkinson, P.; Plumhof, J. D.; Brink, J.; Rastelli, A.; Schmidt, O. G. Universal recovery of the energylevel degeneracy of bright excitons in InGaAs quantum dots without a structure symmetry. Phys. Rev. Lett. 2012, 109, 147401.

(15) Patel, R. B.; Bennett, A. J.; Farrer, I.; Nicoll, C. A.; Ritchie, D. A.; Shields, A. J. Two-photon interference of the emission from electrically tunable remote quantum dots. Nat. Photonics 2010, 4 (9), 632-635.

(16) Zhang, J.; Wildmann, J. S.; Ding, F.; Trotta, R.; Huo, Y.; Zallo, E.; Huber, D.; Rastelli, A.; Schmidt, O. G. High yield and ultrafast sources of electrically triggered entangled-photon pairs based on strain-tunable quantum dots. Nat. Commun. 2015, 6, 10067.

(17) Trotta, R.; Rastelli, A.; Predojević, A., Mitchell, M. W., Eds. Engineering of quantum dot photon sources via electro-elastic fields. In Cham; Springer International Publishing, 2015; pp 277-302.

(18) Akopian, N.; Wang, L.; Rastelli, A.; Schmidt, O. G.; Zwiller, V. Hybrid semiconductor-atomic interface: slowing down single photons from a quantum dot. Nat. Photonics 2011, 5 (4), 230-233.

(19) Zhang, R.; Garner, S. R.; Hau, L. V. Creation of Long-Term Coherent Optical Memory via Controlled Nonlinear Interactions in Bose-Einstein Condensates. Phys. Rev. Lett. 2009, 103 (23), 233602.

(20) Briegel, H.-J.; Dür, W.; Cirac, J. I.; Zoller, P. Quantum repeaters: the role of imperfect local operations in quantum communication. Phys. Rev. Lett. 1998, 81 (26), 5932-5935.

(21) Durnev, M. V.; Vidal, M.; Bouet, L.; Amand, T.; Glazov, M. M.; Ivchenko, E. L.; Zhou, P.; Wang, G.; Mano, T.; Ha, N.; Kuroda, T.; Marie, X.; Sakoda, K.; Urbaszek, B. Magneto-spectroscopy of excited states in charge-tunable GaAs/AlGaAs [111] quantum dots. Phys. Rev. B: Condens. Matter Mater. Phys. 2016, 93 (24), 245412.

(22) Langer, F.; Plischke, D.; Kamp, M.; Höfling, S. Single Photon Emission of a Charge-Tunable GaAs/ $\mathrm{Al}_{0.25} \mathrm{Ga}_{0.75}$ As Droplet Quantum Dot Device. Appl. Phys. Lett. 2014, 105, 81111.

(23) Heyn, C.; Stemmann, A.; Köppen, T.; Strelow, C.; Kipp, T.; Grave, M.; Mendach, S.; Hansen, W. Highly uniform and strain-free GaAs quantum dots fabricated by filling of self-assembled nanoholes. Appl. Phys. Lett. 2009, 94 (18), 183113.

(24) Huo, Y. H.; Rastelli, A.; Schmidt, O. G. Ultra-small excitonic fine structure splitting in highly symmetric quantum dots on GaAs (001) substrate. Appl. Phys. Lett. 2013, 102 (15), 152105.

(25) Huo, Y. H.; Witek, B. J.; Kumar, S.; Cardenas, J. R.; Zhang, J. X.; Akopian, N.; Singh, R.; Zallo, E.; Grifone, R.; Kriegner, D.; Trotta, R.; Ding, F.; Stangl, J.; Zwiller, V.; Bester, G.; Rastelli, A.; Schmidt, O. G. A light-hole exciton in a quantum dot. Nat. Phys. 2013, 10 (1), 46-51. (26) Huo, Y. H.; Křápek, V.; Rastelli, A.; Schmidt, O. G. Volume dependence of excitonic fine structure splitting in geometrically similar quantum dots. Phys. Rev. B: Condens. Matter Mater. Phys. 2014, 90 (4), 041304.

(27) Jahn, J.-P.; Munsch, M.; Béguin, L.; Kuhlmann, A. V.; Renggli, M.; Huo, Y.; Ding, F.; Trotta, R.; Reindl, M.; Schmidt, O. G.; Rastelli, A.; Treutlein, P.; Warburton, R. J. An artificial $\mathrm{Rb}$ atom in a semiconductor with lifetime-limited linewidth. Phys. Rev. B: Condens. Matter Mater. Phys. 2015, 92 (24), 245439.

(28) Huber, D.; Reindl, M.; Huo, Y.; Huang, H.; Wildmann, J. S.; Schmidt, O. G.; Rastelli, A.; Trotta, R. Highly indistinguishable and strongly entangled photons from symmetric GaAs quantum dots. arXiv: 1610.06889, 2016

(29) Kumar, S.; Trotta, R.; Zallo, E.; Plumhof, J. D.; Atkinson, P.; Rastelli, A.; Schmidt, O. G. Strain-induced tuning of the emission wavelength of high quality GaAs/AlGaAs quantum dots in the spectral range of the 87Rb D2 lines. Appl. Phys. Lett. 2011, 99 (16), 161118.
(30) Camacho, R. M.; Pack, M. V.; Howell, J. C.; Schweinsberg, A.; Boyd, R. W. Wide-bandwidth, tunable, multiple-pulse-width optical delays using slow light in cesium vapor. Phys. Rev. Lett. 2007, 98 (15), 153601 .

(31) Siddons, P.; Adams, C. S.; Ge, C.; Hughes, I. G. Absolute absorption on rubidium D lines: comparison between theory and experiment. J. Phys. B: At., Mol. Opt. Phys. 2008, 41 (15), 155004.

(32) Siyushev, P.; Stein, G.; Wrachtrup, J.; Gerhardt, I. Molecular photons interfaced with alkali atoms. Nature 2014, 509 (7498), 6670.

(33) Wildmann, J. S.; Trotta, R; Martín-Sánchez, J.; Zallo, E.; O'Steen, M.; Schmidt, O. G.; Rastelli, A. Atomic clouds as spectrally selective and tunable delay lines for single photons from quantum dots. Phys. Rev. B: Condens. Matter Mater. Phys. 2015, 92 (23), 235306.

(34) Phillips, D. F.; Fleischhauer, A.; Mair, A.; Walsworth, R. L.; Lukin, M. D. Storage of light in atomic vapor. Phys. Rev. Lett. 2001, 86 (5), 783-786.

(35) Trotta, R.; Martín-Sánchez, J.; Wildmann, J. S.; Piredda, G.; Reindl, M.; Schimpf, C.; Zallo, E.; Stroj, S.; Edlinger, J.; Rastelli, A. Wavelength-tunable sources of entangled photons interfaced with atomic vapours. Nat. Commun. 2016, 7, 10375.

(36) Regelman, D. V.; Mizrahi, U.; Gershoni, D.; Ehrenfreund, E.; Schoenfeld, W. V.; Petroff, P. M. Semiconductor quantum dot: A quantum light source of multicolor photons with tunable statistics. Phys. Rev. Lett. 2001, 87 (25), 257401.

(37) Conterio, M. J.; Sköld, N.; Ellis, D. J. P.; Farrer, I.; Ritchie, D. A.; Shields, A. J. A quantum dot single photon source driven by resonant electrical injection. Appl. Phys. Lett. 2013, 103 (16), 10-14.

(38) Dietrich, C. P.; Fiore, A.; Thompson, M. G.; Kamp, M.; Höfling, S. GaAs integrated quantum photonics: Towards compact and multifunctional quantum photonic integrated circuits. Laser Photon. Rev. 2016, 10 (6), 870-894.

(39) Sprague, M. R.; Michelberger, P. S.; Champion, T. F. M.; England, D. G.; Nunn, J.; Jin, X.-M.; Kolthammer, W. S.; Abdolvand, A.; Russell, P. S. J.; Walmsley, I. A. Broadband single-photon-level memory in a hollow-core photonic crystal fibre. Nat. Photonics 2014, 8 (4), 287-291.

(40) Slepkov, A. D.; Bhagwat, A. R.; Venkataraman, V.; Londero, P.; Gaeta, A. L. Spectroscopy of Rb atoms in hollow-core fibers. Phys. Rev. A: At., Mol., Opt. Phys. 2010, 81 (5), 053825.

(41) Bajcsy, M.; Hofferberth, S.; Balic, V.; Peyronel, T.; Hafezi, M.; Zibrov, A. S.; Vuletic, V.; Lukin, M. D. Efficient all-optical switching using slow light within a hollow fiber. Phys. Rev. Lett. 2009, 102 (20), 203902. 